\title{
CONVEX BODIES AND GAUSSIAN PROCESSES
}

\author{
RICHARD A. VITALE
}

Department of Statistics, University of Connecticut, Storrs, CT USA 06269

e-mail: r.vitale@uconn.edu

(Accepted October 11, 2009)

\begin{abstract}
For several decades, the topics of the title have had a fruitful interaction. This survey will describe some of these connections, including the GB/GC classification of convex bodies, Ito-Nisio singularities from a geometric viewpoint, Gaussian representation of intrinsic volumes, the Wills functional in a Gaussian context, and inequalities.
\end{abstract}

Keywords: convex body, Gaussian process, intrinsic volume, random convex body, strong law of large numbers for random convex bodies, Wills functional.

\section{INTRODUCTION}

For several decades, the topics of the title have had a fruitful interaction. This survey will describe some of these connections, including the GB/GC classification of convex bodies, Ito-Nisio singularities from a geometric viewpoint, Gaussian representation of intrinsic volumes, the Wills functional in a Gaussian context, and inequalities. For fuller discussions and references, the interested reader is urged to consult the bibliography.

\section{GEOMETRIC PRELIMINARIES AND NOTATION}

The setting is either finite dimensions or infinite dimensions, that is, $\boldsymbol{R}^{d}$ or $\ell_{2}$. Schneider (1993) gives an excellent treatment of the classical theory of convex bodies. The following items and notation will be appear:

- Convex bodies $\mathscr{K}$ : compact, convex $K, L, \ldots$

- Scaling: $\lambda K=\{\lambda x: x \in K\}$.

- Minkowski addition: $K+L=\{x+y: x \in K, y \in$ $L\}$.

- Closed unit ball: $B, B_{d}$.

- $\lambda$-parallel body: $K+\lambda B$.

- Support function: $h_{K}(x)=\sup _{t \in K}<x, t>$.

- Hausdorff metric:

$$
\begin{aligned}
\rho(K, L) & =\inf \{\lambda>0: K \subseteq L+\lambda B, L \subseteq K+\lambda B\} \\
& =\sup _{\|u\|=1}\left|h_{K}(u)-h_{L}(u)\right| .
\end{aligned}
$$

- Norm: $\|K\|=\max _{x \in K}\|x\|=\max _{\|u\|=1} h_{K}(u)$.

\section{GAUSSIAN PROCESSES WITH ISONORMAL INDEXING}

For background and references on Gaussian processes, one can consult, for example, Lifshits (1995) and Bogachev (1998). We assume throughout a sequence of independent standard (i.e., $N(0,1)$ ) Gaussian random variables:

$$
Z=\left(Z_{1}, Z_{2}, \ldots\right)
$$

For a convex body $K \subset \ell_{2}$ and $t \in K$, we consider the map

$$
t \mapsto X_{t}=<t, Z>=\sum_{i=1}^{\infty} t_{i} Z_{i}
$$

The image is an $N\left(0,\|t\|^{2}\right)$ variable, and the collection $\left\{X_{t}, t \in K\right\}$ is called an isonormally-indexed Gaussian process in view of the isometric-isomorphism:

$$
K \longleftrightarrow\left\{X_{t}, t \in K\right\}
$$

Specifically,

$$
\begin{gathered}
a t+b \hat{t} \leftrightarrow a X_{t}+b X_{\hat{t}} \\
\|t-\hat{t}\|^{2}=E\left(X_{t}-X_{\hat{t}}\right)^{2} .
\end{gathered}
$$

Another key point is the identification

$$
h_{K}(Z)=\sup _{t \in K}<t, Z>=\sup _{t \in K} X_{t} .
$$




\section{LIMIT THEOREMS}

Consider a random convex body $X$, which is a measurable map from a probability space to its space of values endowed with the Hausdorff metric:

$$
X:\{\Omega, \mathscr{F}, P\} \longrightarrow(\mathscr{K}, \rho) .
$$

If $X$ is bounded in expected norm, $E\|X\|<\infty$, then one has an expectation $E X \in \mathscr{K}$, which can be given implicitly in terms of its support function

$$
h_{E X}(\cdot)=E h_{X}(\cdot) .
$$

There is a strong law of large numbers:

Theorem 1 (Artstein and Vitale, 1975) If $X_{1}, X_{2}, \ldots$ are independent and identically distributed random convex bodies with $E\left\|X_{1}\right\|<\infty$, then

$$
\overline{X_{n}}=\frac{X_{1}+X_{2}+\cdots+X_{n}}{n} \stackrel{\text { a.s. }}{\longrightarrow} E X_{1} \text {. }
$$

The formulation of an accompanying central limit theorem takes into account that there is no convenient notion of subtraction for convex bodies, and so the identification with support functions is used:

Theorem 2 (Weil, 1982) If $X_{1}, X_{2}, \ldots$ are iid and $E\|X\|^{2}<\infty$, then $\sqrt{n}\left[h_{\overline{X_{n}}}(u)-h_{E X_{1}}(u)\right]$ converges to a centered Gaussian process with inherited covariance function.

A different kind of limit theorem appears in Bonetti and Vitale (2000).

\section{THE STEINER FORMULA AND INTRINSIC VOLUMES}

The Steiner formula for the volume of the parallel body to a convex body in $\boldsymbol{R}^{d}$ is

$$
\operatorname{vol}_{d}(K+\lambda B)=\sum_{j=0}^{d} \operatorname{vol}_{j}\left(B_{j}\right) \lambda^{j} V_{d-j}(K),
$$

where the constants $V_{j}(K), j=0,1, \ldots, d$ are known as intrinsic volumes.

Following Vitale (1995), we give a derivation of the formula, which also serves to display the nature of the intrinsic volumes: consider iid isotropic line segments $L_{1}, \ldots, L_{n}$, such that $E L_{1}=B_{d}$. By the strong law of large numbers,

$$
(1 / n)\left(L_{1}+\cdots+L_{n}\right) \rightarrow B_{d}
$$

as $n \rightarrow \infty$, and so

$$
\operatorname{vol}_{d}\left[K+(\lambda / n)\left(L_{1}+\cdots+L_{n}\right)\right] \rightarrow \operatorname{vol}_{d}\left(K+\lambda B_{d}\right) .
$$

For one line segment (i.e., $n=1$ ), one has

$$
\operatorname{vol}_{d}\left(K+\lambda L_{1}\right)=\operatorname{vol}_{d}(K)+\lambda\left|L_{1}\right| \cdot \operatorname{vol}_{d-1}\left(\Pi_{L_{1}^{\perp}} K\right),
$$

where $\Pi_{L_{1}^{\perp}}$ signifies projection onto the subspace orthogonal to the one spanned by $L_{1}$. By induction,

$$
\begin{aligned}
& \operatorname{vol}_{d}\left[K+(\lambda / n)\left(L_{1}+\cdots+L_{n}\right)\right]= \\
& \quad \sum_{\substack{S \subseteq\{1,2, \ldots, n\} \\
0 \leq S S \mid \leq d}}(\lambda / n)^{|S|} \operatorname{vol}_{|S|}\left(L_{S}\right) \operatorname{vol}_{d-|S|}\left(\Pi_{L_{S}^{\perp}} K\right),
\end{aligned}
$$

where $L_{S}=\sum_{i \in S} L_{i}$. This can be re-expressed as

$$
\operatorname{vol}_{d}\left[K+(\lambda / n)\left(L_{1}+\cdots+L_{n}\right)\right]=\sum_{j=0}^{d} \frac{\left(\begin{array}{c}
n \\
j
\end{array}\right)}{n^{j}} \lambda^{j} U_{j n},
$$

where

$$
U_{j n}=\frac{1}{\left(\begin{array}{c}
n \\
j
\end{array}\right)} \sum_{|S|=j} \operatorname{vol}_{j}\left(L_{S}\right) \operatorname{vol}_{d-j}\left(\Pi_{L_{S}^{\perp}} K\right)
$$

has the form of a $U$-statistic. Then

$$
\begin{aligned}
\operatorname{vol}_{d}\left(K+\lambda B_{d}\right) & =\lim _{n} \operatorname{vol}_{d}\left[K+(\lambda / n)\left(L_{1}+\cdots+L_{n}\right)\right] \\
& =\lim _{n} \sum_{j=0}^{d} \frac{\left(\begin{array}{c}
n \\
j
\end{array}\right)}{n^{j}} \lambda^{j} U_{j n} \\
& =\sum_{j=0}^{d} \frac{\lambda^{j}}{j !} \lim _{n} U_{j n} \\
& =\sum_{j=0}^{d} \frac{\lambda^{j}}{j !} c_{d-j} E \operatorname{vol}_{d-j}\left(\Pi_{d-j} K\right) \\
& =\sum_{j=0}^{d} \operatorname{vol}_{j}\left(B_{j}\right) \lambda^{j} V_{d-j}(K),
\end{aligned}
$$

where $\Pi_{j}$ signifies projection onto a random subspace of dimension $j$ and

$$
V_{j}(K)=\left(\begin{array}{c}
d \\
j
\end{array}\right) \frac{\operatorname{vol}_{d}\left(B_{d}\right)}{\operatorname{vol}_{j}\left(B_{j}\right) \operatorname{vol}_{d-j}\left(B_{d-j}\right)} E \operatorname{vol}_{j}\left(\Pi_{j} K\right) .
$$

A Gaussian version, shown below in Eq. 2, follows from noticing that, in Eq. 1, a key property is that, for an independent, random orthogonal $O$,

$$
\Pi_{j} O \stackrel{\mathrm{d}}{=} \Pi_{j} .
$$


It is also true that

$$
Z_{[j, d]} O \stackrel{\mathrm{d}}{=} Z_{[j, d]},
$$

where $Z_{[j, d]}$, is a $j \times d$ matrix of independent $N(0,1)$ variables. This can be used (Vitale, 2008) to show

$$
V_{j}(K)=\frac{(2 \pi)^{j / 2} E \operatorname{vol}_{j}\left(Z_{[j, d]} K\right)}{j ! \operatorname{vol}_{j}\left(B_{j}\right)} .
$$

Next we identify some of the intrinsic volumes:

$$
\begin{aligned}
& V_{0}(K)=1 \\
& V_{1}(K)=\text { intrinsic width } \\
&=\sqrt{2 \pi} E h_{K}(Z)=\sqrt{2 \pi} E \sup _{t \in K} X_{t} \\
& \vdots \\
& V_{d-1}(K)=1 / 2 \cdot \text { surface area of } K \\
& V_{d}(K)=d \text {-dimensional volume of } K \\
& V_{j}(K)=0 \text { for } j>d \\
& V_{j}\left(\prod_{1}^{n}\left[a_{i}, b_{i}\right]\right)=\sum_{i_{1}<i_{2}<\cdots<i_{j}}\left(b_{i_{1}}-a_{i_{1}}\right) \cdots\left(b_{i_{j}}-a_{i_{j}}\right) \\
& V_{1}\left(B_{d}\right) \sim \sqrt{2 \pi d} . \\
& \text { EXTENSION OF INTRINSIC } \\
& \text { VOLUMES TO CONVEX BODIES } \\
& \text { IN } \ell_{2}
\end{aligned}
$$

\section{EXTENSION OF INTRINSIC VOLUMES TO CONVEX BODIES \\ IN $\ell_{2}$}

The extension of intrinsic volumes to convex bodies in Hilbert space and specifically to $\ell_{2}$ was undertaken by Sudakov (1971) and Chevet (1976). To begin, let us identify the following collections:

$$
\begin{aligned}
\mathscr{K}_{\mathrm{d}}= & \text { convex bodies in } \boldsymbol{R}^{d} \\
\mathscr{K}= & \text { convex bodies in } \ell_{2} \\
\mathscr{K}_{\mathrm{FD}}= & \text { finite-dimensional convex bodies } \\
& \text { in } \ell_{2} .
\end{aligned}
$$

In view of the monotonicity of the intrinsic volumes under set inclusion, it is natural to extend them to infinite dimensional convex bodies as follows: for arbitrary $K \in \mathscr{K}$, define

$$
\begin{aligned}
V_{j}(K) & =\sup \left\{V_{j}(\widehat{K}): \widehat{K} \subseteq K, \widehat{K} \in \mathscr{K}_{\mathrm{FD}}\right\} \\
\mathscr{K}_{\mathrm{GB}} & =\left\{K \in \mathscr{K}: V_{1}(K)<\infty\right\} .
\end{aligned}
$$

GB stands for "Gaussian Bounded" (Dudley, 1967) and refers to the following identification.

Theorem $3 \quad K \in \mathscr{K}_{\mathrm{GB}} \Longleftrightarrow\left\{X_{t}, t \in K\right\}$ is an almost-surely bounded Gaussian process. That is $P\left(\sup _{t \in K_{0}} X_{t}<\infty\right)=1$ for any denumerable subset $K_{0} \subset K$.
The following also hold:

1. $\mathscr{K}_{\mathrm{FD}} \subset \mathscr{K}_{\mathrm{GB}} \subset \mathscr{K}$.

2. $K \in \mathscr{K}_{\mathrm{GB}} \Rightarrow V_{j}(K)<\infty, \quad j=2,3, \ldots$

3. $K \in \mathscr{K}_{\mathrm{GB}} \Rightarrow V_{j}(K)=\frac{(2 \pi)^{j / 2} E \operatorname{vol}_{j}\left(Z_{[j, \infty]} K\right)}{j ! \operatorname{vol}_{j}\left(B_{j}\right)}$, where $Z_{[j, \infty]}$ is a $j \times \infty$ matrix of independent $N(0,1)$ variables. Equivalently (Tsirel'son, 1985),

$$
V_{j}(K)=\frac{(2 \pi)^{j / 2} E \operatorname{vol}_{j}\left(\left\{\left(X_{t}^{1}, X_{t}^{2}, \ldots, X_{t}^{j}\right), t \in K\right\}\right)}{j ! \operatorname{vol}_{j}\left(B_{j}\right)} .
$$

Some canonical cases are given in the next example.

Example Given a decreasing sequence of positive constants $\left\{a_{n}\right\}$ and an orthonormal set $\left\{e_{n}, n=\right.$ $1,2, \ldots\}$, set

$$
K=\overline{\operatorname{conv}}\left\{a_{n} e_{n}, n=1,2, \ldots\right\} .
$$

Then

$$
\begin{aligned}
K \in \mathscr{K} & \Longleftrightarrow a_{n} \downarrow 0, \\
K \in \mathscr{K}_{\mathrm{FD}} & \Longleftrightarrow a_{n}=0 \text { eventually, } \\
K \in \mathscr{K}_{\mathrm{GB}} & \Longleftrightarrow a_{n}=\mathscr{O}\left[(\log n)^{-1 / 2}\right] .
\end{aligned}
$$

Example An example of an infinite-dimensional convex body that has no finite-dimensional analogue is as follows. Consider a map

$$
f:[0,1] \rightarrow \mathscr{H}(\text { Hilbert space })
$$

that satisfies

1. $0 \leq x_{1} \leq x_{2} \leq x_{3} \leq x_{4} \leq 1 \Rightarrow$

$$
\left[f\left(x_{2}\right)-f\left(x_{1}\right)\right] \perp\left[f\left(x_{4}\right)-f\left(x_{3}\right)\right]
$$

2. $\left\|f\left(x_{2}\right)-f\left(x_{1}\right)\right\|^{2}=\left|x_{2}-x_{1}\right|$ for all $0 \leq x_{1} \leq x_{2} \leq 1$.

The associated Brownian Motion Body is defined to be

$$
\overline{\operatorname{conv}}\{f([0,1])\} \subset \mathscr{H} .
$$

All Brownian motion bodies are the same, of course, up to an isometry. A particular realization in $L^{2}[0,1]$ is

$$
\left\{g:[0,1] \rightarrow \boldsymbol{R}^{1} \mid 0 \leq g \leq 1, g \uparrow\right\} .
$$

Theorem 4 (Gao and Vitale, 2001)

$$
V_{j}(B M B)=\frac{\operatorname{vol}_{j}\left(B_{j}\right)}{j !} \quad j=1,2, \ldots
$$




\section{SINGULARITIES}

Although intrinsic volumes are defined, and finite, for all GB convex bodies, they are not continuous. That is, it is possible to have GB bodies with $K_{n} \rightarrow K$, but $V_{j}\left(K_{n}\right) \nrightarrow V_{j}(K)$. In particular, one can have

$$
K_{n} \downarrow\{p\}, \text { but } V_{1}\left(K_{n}\right) \downarrow 0=V_{1}(\{p\}) .
$$

This leads to the following definition.

Definition $t^{*} \in K \in \mathscr{K}_{\mathrm{GB}}$ is a singularity of $K$ if

$$
V_{1}\left(K \cap B\left(t^{*}, \varepsilon\right)\right) \downarrow 0 \quad \text { as } \varepsilon \rightarrow 0 .
$$

Definition $\quad \mathscr{K}_{\mathrm{GC}}=\left\{K \in \mathscr{K}_{\mathrm{GB}}: K\right.$ has no singularities\}.

One has

$$
\mathscr{K}_{\mathrm{FD}} \subset \mathscr{K}_{\mathrm{GC}} \subset \mathscr{K}_{\mathrm{GB}} \subset \mathscr{K} .
$$

GC stands for "Gaussian Continuous" (Dudley, 1967), and the following gives the connection.

Theorem $5 K \in \mathscr{K}_{\mathrm{GC}} \Longleftrightarrow\left\{X_{t}, t \in K\right\}$ is an almostsurely continuous Gaussian process. That is $t_{n} \rightarrow t \Rightarrow$ $X_{t_{n}} \rightarrow X_{t}$ almost-surely.

Example (continued)

$$
\begin{gathered}
K=\overline{\operatorname{conv}}\left\{a_{n} e_{n}, n=1,2, \ldots\right\} \in \mathscr{K}_{\mathrm{GC}} \\
\Longleftrightarrow a_{n}=o\left[(\log n)^{-1 / 2}\right] .
\end{gathered}
$$

\section{ITO-NISIO THEORY}

Theorem 6 (Ito and Nisio, 1969) Suppose that $t^{*} \in$ $K \in \mathscr{K}_{\mathrm{GB}}$. The oscillation of $X$ at $t^{*}$, given by

$$
0 \leq 2 \cdot \operatorname{osc}\left(t^{*}\right)=\lim _{\varepsilon \downarrow 0}\left[\sup _{t \in K \cap B\left(t^{*}, \varepsilon\right)} X_{t}-\inf _{t \in K \cap B\left(t^{*}, \varepsilon\right)} X_{t}\right],
$$

is almost surely constant. Further, $\operatorname{osc}\left(t^{*}\right)>0 \Longleftrightarrow t^{*}$ is a singularity of $K$.

The following elaborates this observation.

Theorem 7 (Vitale, 2001) Suppose that osc $\left(t^{*}\right)>0$. Then

1. $\operatorname{osc}\left(t^{*}\right) \stackrel{\text { a.s. }}{=} \frac{1}{\sqrt{2 \pi}} \lim _{\mathcal{\varepsilon} \downarrow 0} V_{1}\left(K \cap B\left(t^{*}, \varepsilon\right)\right)$.

2. For each $j$,

$$
\lim _{\varepsilon \downarrow 0} V_{j}\left(K \cap B\left(t^{*}, \varepsilon\right)\right)>0 .
$$

3. $K \cap B\left(t^{*}, 0+\right) \approx \frac{1}{\sqrt{2 \cdot \pi \cdot \infty}} B_{\infty}\left(t^{*}, \operatorname{osc}\left(t^{*}\right)\right)$ in the sense that for each $j$, the limit in Eq. 3 is equal to

$$
\lim _{d \rightarrow \infty} V_{j}\left(\frac{1}{\sqrt{2 \cdot \pi \cdot d}} B\left(t^{*}, \operatorname{osc}\left(t^{*}\right)\right)\right)
$$

(both being $\left.\frac{\operatorname{osc} j\left(t^{*}\right)}{j !}\right)$.

4. Define $\operatorname{osc}(K)=\sup \left\{E \operatorname{osc}\left(t^{*}\right): t^{*} \in K\right\}$. Then

$$
\operatorname{osc}(K)=\lim _{j \rightarrow \infty} \frac{(j+1) V_{j+1}(K)}{V_{j}(K)} .
$$

\section{THE WILLS FUNCTIONAL AND BOUNDS FOR GAUSSIAN PROCESSES}

In the context of a question in lattice point enumeration, Wills (1973) defined the following functional. It has come to play an important role in the connection between the theories of convex bodies and Gaussian processes.

$$
W(K)=\int_{R^{d}} \frac{1}{(2 \pi)^{d / 2}} \mathrm{e}^{-\frac{1}{2} \operatorname{dist}^{2}(x, K)} \mathrm{d} x .
$$

An alternate representation can be derived as follows:

$$
\begin{aligned}
W(K) & =\frac{1}{(2 \pi)^{d / 2}} \int_{R^{d}} P(\operatorname{dist}(x, K) \leq \Lambda) \mathrm{d} x, \\
& =\frac{1}{(2 \pi)^{d / 2}} \int_{R^{d}} E 1(\operatorname{dist}(x, K) \leq \Lambda) \mathrm{d} x \\
& =\frac{1}{(2 \pi)^{d / 2}} E \int_{R^{d}} 1(\operatorname{dist}(x, K) \leq \Lambda) \mathrm{d} x \\
& =\frac{1}{(2 \pi)^{d / 2}} E \operatorname{vol}(K+\Lambda B) . \\
& =\frac{1}{(2 \pi)^{d / 2}} E\left[\sum_{i=0}^{d} \operatorname{vol}_{i}\left(B_{i}\right) \Lambda^{i} V_{d-i}(K)\right] \\
& =\frac{1}{(2 \pi)^{d / 2}} \sum_{i=0}^{d} \operatorname{vol}_{i}\left(B_{i}\right)\left[E \Lambda^{i}\right] V_{d-i}(K) \\
& =\frac{1}{(2 \pi)^{d / 2}} \sum_{i=0}^{d} \operatorname{vol}_{i}\left(B_{i}\right)\left[\frac{(2 \pi)^{i / 2}}{\operatorname{vol}_{i}\left(B_{i}\right)}\right] V_{d-i}(K) \\
& =\sum_{j=0}^{d} \frac{1}{(2 \pi)^{j / 2}} V_{j}(K) .
\end{aligned}
$$


A second alternate representation proceeds as follows:

$$
\begin{aligned}
W(K) & =\int_{R^{d}} \frac{1}{(2 \pi)^{d / 2}} \mathrm{e}^{-\frac{1}{2} \operatorname{dist}^{2}(x, K)} \mathrm{d} x \\
& =\int_{R^{d}} \frac{1}{(2 \pi)^{d / 2}} \mathrm{e}^{-\frac{1}{2} \inf _{t \in K}\|x-t\|^{2}} \mathrm{~d} x \\
& =\int_{R^{d}} \mathrm{e}^{\sup _{t \in K}\left[<x, t>-\frac{1}{2}\|t\|^{2}\right]} \frac{1}{(2 \pi)^{d / 2}} \mathrm{e}^{-\frac{1}{2}\|x\|^{2}} \mathrm{~d} x \\
& =E \mathrm{e}^{\sup _{t \in K}\left[X_{t}-\frac{1}{2} \sigma_{t}^{2}\right]} .
\end{aligned}
$$

One then has

$$
E \mathrm{e}^{\sup _{t \in K}\left[X_{t}-\frac{1}{2} \sigma_{t}^{2}\right]}=\sum_{j=0}^{d} \frac{1}{(2 \pi)^{j / 2}} V_{j}(K) .
$$

This can be extended by writing $r K, r>0$, in place of $K$, which itself can be taken to be an element of $\mathscr{K}_{\mathrm{GB}}$ :

$$
E \mathrm{e}^{\sup _{t \in K}\left[r X_{t}-\frac{1}{2} r^{2} \sigma_{t}^{2}\right]}=\sum_{j=0}^{\infty}\left(\frac{r}{\sqrt{2 \pi}}\right)^{j} V_{j}(K) .
$$

The Alexandrov-Fenchel inequality (Schneider, 1993) implies that

$$
V_{j}(K) \leq \frac{1}{j !} V_{1}^{j}(K)=\frac{1}{j !}\left[\sqrt{2 \pi} E \sup _{t \in K} X_{t}\right]^{j},
$$

and hence

$$
\sum_{j=0}^{\infty}\left(\frac{r}{\sqrt{2 \pi}}\right)^{j} V_{j}(K) \leq \mathrm{e}^{r E \sup _{t \in K} X_{t}} .
$$

Thus we have shown the following:

Theorem 8 (Tsirel'son, 1985; Vitale, 1996, 2001 ) If $\left\{X_{t}, t \in K\right\}$ is a mean-zero, bounded Gaussian process, then

$$
E \mathrm{e}^{\sup _{t}\left\{X_{t}-(1 / 2) E X_{t}^{2}\right\}} \leq \mathrm{e}^{E \sup _{t} X_{t}} .
$$

An immediate consequence is a deviation bound:

\section{Theorem 9 (Pisier, 1986; Vitale, 1996, 1999)}

$$
P\left(\sup _{t} X_{t}-E \sup _{t} X_{t} \geq a\right) \leq \exp \left[-(1 / 2)\left(a^{2} / \sigma^{2}\right)\right] \text {. }
$$

Proof: Set $\sigma^{2}=\sup _{t \in K} \sigma_{t}^{2}$. It is direct to show

$$
E \mathrm{e}^{r\left[\sup _{t \in K} X_{t}-E \sup _{t \in K} X_{t}\right]} \leq \mathrm{e}^{\frac{1}{2} r^{2} \sigma^{2}} .
$$

Then

$$
\begin{aligned}
P\left(\sup _{t \in K} X_{t}-E \sup _{t \in K} X_{t} \geq a\right)= \\
\quad=P\left(r\left[\sup _{t \in K} X_{t}-E \sup _{t \in K} X_{t}\right] \geq r a\right) \\
\quad=P\left(\mathrm{e}^{r\left[\sup _{t \in K} X_{t}-E \sup _{t \in K} X_{t}\right]} \geq \mathrm{e}^{r a}\right) \\
\leq E \mathrm{e}^{r\left[\sup _{t \in K} X_{t}-E \sup _{t \in K} X_{t}\right]} \mathrm{e}^{-r a} \\
\leq \mathrm{e}^{\frac{1}{2} r^{2} \sigma^{2}-r a}
\end{aligned}
$$

which is minimized at $r=a / \sigma^{2}$, and the assertion follows.

\section{CONCLUSION}

We have surveyed topics that combine elements of the theory of convex bodies and Gaussian processes.

\section{ACKNOWLEDGMENT}

An earlier version of this paper appeared in the accompanying Proceedings of the 10th European Congress of Stereology and Image Analysis (Milan, 2009).

\section{REFERENCES}

Artstein Z, Vitale RA (1975). A strong law of large numbers for random compact sets. Ann Probab 3:879-82.

Bogachev VI (1998). Gaussian Measures. Providence: American Mathematical Society.

Bonetti M, Vitale RA (2000). Asymptotic behavior of a setstatistic. Discrete Comput Geom 23:333-41.

Chevet S (1976). Processus Gaussiens et volumes mixtes. Z Wahr Verw Geb 36:47-65.

Dudley RM (1967). The sizes of compact subsets of Hilbert space and continuity of Gaussian processes. J Funct Anal 1:290-330.

Gao F, Vitale RA (2001). Intrinsic volumes of the Brownian motion body. Discrete Comput Geom 26:41-50.

Ito K, Nisio M (1969). On the oscillation functions of Gaussian processes. Math Scand 22:209-23.

Lifshits MA (1995). Gaussian Random Functions. Dordrecht: Kluwer.

Pisier G (1986). Probabilistic methods in the geometry of Banach spaces. In: Letta G, Pratelli M, eds., Probability and analysis. Lect Notes Math 1206:167-241.

Schneider R (1993). Convex Bodies: the Brunn-Minkowski Theory. New York: Cambridge University Press.

Sudakov VN (1971). Gaussian random processes and measures of solid angles in a Hilbert space. Dokl Akad Nauk SSSR 197:43-5 (in Russian); English translation in Soviet Math Dokl (1971) 12:412-5.

Tsirel'son BS (1985). A geometric approach to maximum likelihood estimation for infinite-dimensional Gaussian location II. Theor Probab Appl 30:820-8. 
Vitale RA (1995). On the volume of parallel bodies: a probabilistic derivation of the Steiner formula. Adv Appl Probab 27:97-101.

Vitale RA (1996). The Wills functional and Gaussian processes. Ann Probab 24:2172-8.

Vitale RA (1999). A log-concavity proof for a Gaussian exponential bound. In: Hill TP, Houdré C, eds., Advances in Stochastic Inequalities (Contemporary Math., vol. 234), 209-12. Providence: American Mathematical Society.
Vitale RA (2001). Intrinsic volumes and Gaussian processes. Adv Appl Probab 33:354-64.

Vitale RA (2008). On the Gaussian representation of intrinsic volumes. Stat Probab Lett 78:1246-9.

Weil W (1982). An application of the central limit for Banach-space-valued random variables to the theory of random sets. Probab Theory Rel 60:203-8.

Wills JM (1973). Zum Gitterpunktanzahl konvexer Mengen. Elem Math 28:57-63. 